\title{
Approche écosystémique et fondements de l'intervention éducative précoce en milieux socio- économiques faibles. Les conditions de la résilience scolaire
}

François Larose
Université de Sherbrooke
Bernard Terrisse
Université du Québec à Montréal
Yves Lenoir
Université de Sherbrooke
Johanne Bédard
Université de Montréal

Résumé

Dans cet article, nous faisons état du rôle que l'adoption d'un discours fondé sur le modèle écosystémique a joué sur le plan de l'évolution des pratiques d'intervention éducative précoce auprès de l'enfant de milieu socio-économique faible et de sa famille. Nous situons les limites des interventions qui s'y réfèrent en démontrant la variabilité des fondements épistémologiques qui les orientent et leur impact spécifique sur la conception du rapport de l'enfant aux systèmes dans lesquels il transite. Nous terminons en identifiant les obstacles au développement d'une intervention de réseau, de nature écosystémique, fondée sur une lecture constructiviste du développement, notamment dans la structure même des curriculums préscolaires ainsi que dans la lecture que les intervenants font de la compétence de l'enfant de mséf ainsi que de celle de sa famille.

\begin{abstract}
In this paper we consider the evolution of the child in the low socioeconomic status family in relation to the Bronfenbrenner ecosystemic model. Conceptualy we find that the various epistemological stances underlying so-called ecosystemic interventions represent

François Larose est Professeur, Faculté d'éducationUniversité de Sherbrooke: CRIE/ CRIFPE. Bernard Terrisse est Professeur, Faculté d'éducation à l'Université du Québec à Montréal : GREASS/CRIE. Yves Lenoir est Professeur, Faculté d'éducationUniversité de Sherbrooke : CRIE/CRIFPE. Johanne Bédard est. Professeure, Faculté des sciences de l'éducation à l'Université de Montréal : CRIE/CRIFPE.
\end{abstract}


quite diverse concepts about the relationship between children and the systems in which they interact. If behaviorist approaches are still frequent within the universe of ecosystemic models, the development of constructivist-oriented tendencies in regard to the child-adults and child-systems relationships opens up the way towards research and intervention oriented towards children's and families' resilience. We finish the paper identifying some major obstacles to intervention based on patterns of social networks, ecosystemic in essence and strongly based on a constructivist orientation.

\section{Introduction}

Si l'approche écosystémique sert de cadre de référence conceptuel à une bonne partie des discours portant sur l'intervention éducative auprès de l'enfant d'âge préscolaire et de sa famille, du moins en Amérique du Nord, les lectures qui sont faites des implications d'une telle perspective sont diverses, voire contradictoires. D'une option de type dogmatique, impliquant la reproduction du modèle de Bronfenbrenner (1979) et son «plaquage » sur une lecture particulière du réel, à la référence au dit modèle en tant que justificatif de l'ensemble des angles d'approche de l'intervention psychosociale auprès du petit-enfant ou de l'enfant, on trouve un peu de tout.

D'une façon plus générale, les perspectives epistémologiques qui fondent les recherches se réclamant d'une approche écologique ou écosystémique sont variables. Ces variations sont à l'origine de positions potentiellement réductionnistes, notamment en ce qui concerne la définition de l'écosystème dans lequel l'enfant se développe et avec lequel il interagit. Elles reflètent aussi divers degrés de déterminisme dans la conception du développement psychologique et social de l'individu.

Dans cet article, nous ne reviendrons que brièvement sur les caractéristiques générales du modèle de Bronfenbrenner (1979). Nous centrerons plutôt notre propos sur l'impact que l'adoption d'une perspective constructiviste du développement peut avoir sur la lecture faite par le chercheur ou la practicienne/le praticien à propos des caractéristiques de la relation individu-environnement. Nous soulignerons l'intérêt de la prise en considération du concept d'interaction sociale dans une perspective constructiviste en rapport avec le développement d'une perspective dynamique des systèmes. Nous terminerons notre étude par une prise de position de type relativiste par rapport aux divers sens que peut prendre un discours adoptant une perspective écosystémique au regard du construit de résilience. 


\section{L'approche écosystémique: du déterminisme béhavioriste au relativisme constructiviste}

Dans un article faisant une revue relativement extensive de la documentation scientifique portant sur les recherches qui se réfèrent au modèle de Bronfenbrenner (1979) en éducation spécialisée, Sontag (1996) constatait que, bien que plusieurs d'entre elles se soient référées au paradigme « écologique », elles tendent à le faire de façon réductionniste. L'écosystème de l'enfant y est défini, le plus souvent, de façon additive. La centration se fait d'abord sur les interactions de l'enfant avec les composantes de son environnement social dans le contexte scolaire puis avec l'environnement familial (Tyler \& Jones, 2000). Peu d'études envisagent l'analyse des relations de l'enfant avec son environnement de façon holiste, les composantes ontosystémiques, microsystémiques et mésosystémiques étant conçues de façon interactive et intégrée grâce à la centration sur une composante dynamique: le sujet ou, si on préfère, l'enfant.

Cette dimension réductionniste, qui considère la relation invididuenvironnement sous l'angle du déterminisme environnemental, se retrouve de façon particulièrement marquée en Amérique du Nord. Les études européennes, pour leur part, tendent plus fréquemment à se centrer sur la fonction dynamique du sujet. À l'origine de ces tendances différenciées se trouve sans doute l'influence respective de la psychologie du comportement en Amérique du Nord et celle de la psychologie génétique piagétienne, mais aussi de l'école française, représentée entre autre par Wallon (1938), en Europe. Dans un cas l'individu est « objet «, dans l'autre il est « sujet». Or un objet épistémique ne se développe qu'en conséquence d'une action alors qu'un sujet se construit, généralement dans l'interaction sociale (Lenoir, Larose, \& Hébert, 2000).

Un réductionnisme relatif est inévitable lorsque la perspective écosystémique est considérée sous l'angle spécifique de la psychologie scolaire. Il faut noter que nous limiterons notre propos à l'Amérique du Nord puisque les critères de qualification et d'exercice du rôle de psychologue scolaire, ainsi que le cadre dans lequel s'exerce cette profession, y sont distincts de ceux qui prévalent en France, par exemple. Au Canada, le psychologue scolaire est un psychologue au sens corporatif du terme. Il détient une formation disciplinaire complète de psychologue et intervient en tant que professionnel dans un champ distinct de celui de l'éducateur ou de l'enseignant. En Amérique du Nord, la psychologie scolaire n'existe qu'en fonction d'une définition contextuelle excessivement restreinte, l'adaptation des conduites sociales et, à moindre titre, des conditions favorisant l'adaptation scolaire de l'enfant, à l'intérieur du contexte scolaire lui-même. L'intervention en 
psychologie scolaire est donc généralement plus une intervention curative ou thérapeutique que préventive.

Le modèle de Bronfenbrenner (1979) est, à l'origine, centré sur la relation entre l'enfant (ou l'individu en développement) et des environnements distincts mais dont la complémentarité est assurée par la médiation du sujet. Il s'agit d'un modèle, non pas du développement de l'individu, mais plutôt des contextes dans lesquels ce développement se produit et où il effectue des transactions et des transitions. Cela étant, les environnements ou les contextes peuvent être considérés de façon plus ou moins cloisonnée, comme il est possible de les voir en tant que zone de contextualisation d'une même réalité.

Dans une lecture simplifiée de la dynamique développementale en perspective piagétienne, l'enfant ou l'individu considéré en tant qu'objet assimile l'information qu'un environnement lui fournit et s'accommode au contexte. L'adaptation demeure multiple, contextuelle, et, en conséquence, ne peut être considérée en tant que caractéristique de la structure de l'individu. Dans la même perspective, mais en considérant l'enfant en tant que sujet, son interaction avec chacun des univers contextuels composant le mésosystème mène à une dynamique d'adaptation proactive qui, à son tour, permet l'unification de ces univers au travers du développement d'une personnalité intégrée (Rosenbaum \& Dyckman, 1995). Dans les deux cas, le modèle écosystémique permet une lecture utile des interactions de l'enfant avec son environnement.

Dans le premier cas, l'action éducative, scolaire ou non, à caractère préventif ou curatif, peut être conçue d'une façon qui exclue l'action de l'enfant mais qui en favorise l'adaptation de façon externe. Le contrôle des contingences environnementales propres à un microsystème, ou l'action sur ces dernières, permettra l'adaptation appropriée de l'individu, cette adaptation étant évaluée selon son degré d'adéquation des conduites par rapport à un ensemble de normes de référence qui sont relativement aisément définissables. Nous avons ici une lecture assez conforme à la définition d'une écologie du développement qui s'identifie à une écologie du comportement (Laird, Pettit, Dodge, \& Bates, 1998; Lucyshyn, Albin, \& Nixon, 1997; Ollendick \& King, 1999).

Dans le second cas l'intervention éducative est conçue en tant que médiation de la part de l'intervenant entre l'enfant et l'ensemble des vecteurs de représentations qui composent son environnement. L'enfant est constructeur de représentations dans son action contextualisée sur chacun des microsystèmes dans lesquels il évolue. Il est constructeur de sens et cherche la généralité des savoirs d'action qu'il construit, il est générateur de compétences utilisables à bon escient, dans les limites qu'imposent les contraintes maturationelles liées à son développement, au sens de Rey (1996). Encore faut-il 
que l'environnement offre des situations de déséquilibre engendrant le stress (life events) le forcant à développer des stratégies d'adaptation (coping). S'il sait utiliser les facteurs de protection présents dans son environnement, il développera des compétences utilisables dans un continuum temporel.

Dans le cadre des transitions qui marquent son développement, l'enfant apprend à distinguer le général, où il construit des compétences transversales ou génératives, du particulier où il bâtit des compétences contextualisées ou dites génériques. En tant que sujet, l'enfant est conçu comme étant susceptible, potentiellement, d'engendrer une infinité de conduites adéquates à une infinité de situations nouvelles, en les adaptant et en les transférant de façon contextualisée à d'autres situations lorsque cela s'avère pertinent. Cette seconde conception de la relation individu environnement correspond à une épistémologie du développement de type constructiviste (McMillen, 1999; Miltenburg \& Singer, 2000).

\section{Les deux lectures d'une approche écosystémique dans le contexte de l'intervention précoce}

Il est apparemment plus difficile d'envisager une définition restrictive de l'écosystème de l'enfant dans le cadre de l'intervention précoce comme le suggèrent plusieurs études récentes. En effet, l'intervention éducative auprès de l'enfant d'âge préscolaire et de sa famille tire sa justification de la notion de risque et de celle de prévention, donc d'une intervention préalable, extrascolaire ou «préscolaire », sur une probabilité appréhendée de mésadaptation de l'enfant à l'environnement social au moment de sa scolarisation (Terrisse, Lefebvre, Larose, \& Martinet, 2000). Une conception étroite, restrictive de la lecture du sens donné à la relation de l'enfant au mésosystème mène à une forme d'additivité que maints auteurs justifient en considérant ce dernier comme une zone de coexistence de plusieurs microsystèmes, les environnements mentionnés étant pondérés de façon équivalente à partir du critère de proximité des interactions de l'enfant avec chaque milieu (Mortimer \& Shanahan, 1994).

Bien que conforme sur le plan théorique avec la définition des niveaux d'interaction enfant-milieu que le modèle écologique du développement propose, cette conception « additive » comporte des risques. Dans une conception additive du mésosystème, l'un des microsystèmes, généralement l'environnement familial, peut être considéré en tant que vecteur de risque alors que le second, par exemple, la mesure d'intervention socio-éducative précoce, sera considéré en se en tant que facteur de protection (Terrisse, 2000).

Ainsi, les déficits en tant qu'effet de l'exposition de l'enfant à des pratiques éducatives familiales inappropriées ou à des stresseurs 
environnementaux indus seront compensés en agissant sur une structure environnementale tierce, la mesure socio-éducative précoce. En corollaire, il est possible d'agir sur les facteurs de risque auxquels l'enfant est exposé en aidant les parents à prendre conscience d'attitudes et de pratiques éducatives considérées comme plus favorables que d'autres à l'adaptation de l'enfant (cf. figure 1).

\section{Figure 1}

Modélisation de l'intervention précoce dans une perspective additive, béhavioriste: cloisonnement des microsystèmes

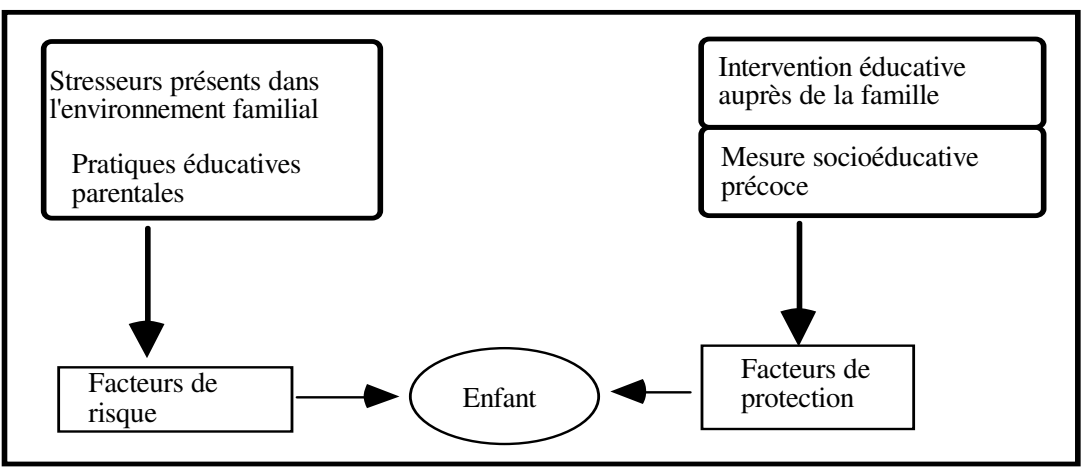

Dans une perspective de type constructiviste une analyse des cohérences des représentations construites par l'enfant ainsi que de celles propres aux diverses catégories d'acteurs de son milieu doit être effectuée. Par la suite, les

cohérences entre ces représentations et les attitudes et conduites des individus doivent être identifiées. Cette identification inclu l'enfant et les acteurs des divers milieux qu'il fréquente en contexte d'interactions situées (Au, 1998; Palincsar, 1998). Cette identification implique la reconnaissance préalable de compétences chez l'ensemble des acteurs des milieux formant le microsystème familial ainsi que les composantes du mésosystème de référence de l'enfant (Holloway, Rambaud, Fuller, \& Eggerspierola, 1995). Cela étant, un système particulier ne peut être considéré dans sa globalité, en tant que vecteur unique de facteurs de risque et, un autre, en tant que porteur exclusif de facteurs de protection. De la même façon, les structures propres aux interactions entre l'enfant et son environnement ne peuvent être considérées en tant que données stables mais doivent être envisagées en tant que phénomènes dynamiques donc qualitativement «mouvants», évoluant dans un chronosystème (cf. figure 2). 
L'approche écosystémique, lorsqu'elle s'applique à l'univers de l'intervention éducative précoce auprès du jeune enfant et de sa famille, en particulier lorsqu'elle repose sur des prémisses épistémologiques de type constructiviste, renvoie le plus souvent au construit de résilience. Une revue de la documentation scientifique portant sur le concept d'intervention dans un paradigme ecosystémique confirme d'ailleurs le caractère intrinsèquement intégré de cette notion et de celle de résilience (Howard, Dryden, \& Johnson, 1999; Howard \& Johnson, 2000; Stoiber \& Good, 1998; Waller, 2001; Walsh, 1996).

Figure 2

Modélisation de l'intervention précoce dans une perspective interactive: décloisonnement des systèmes et complémentarité des dynamiques

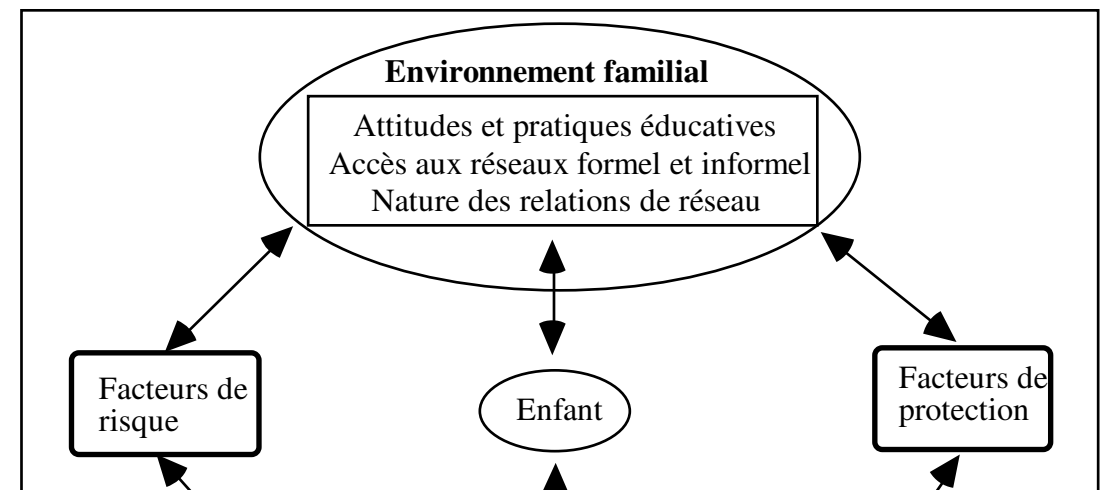

Par conséquent, il est pertinent de se pencher sur les concepts de risque, de résilience et d'intervention précoce pour mieux situer les différents 
profils qu'adoptent les pratiques d'intervention socio-éducative auprès de l'enfant et de la famille selon leurs fondements épistémologiques.

\section{L'intervention précoce}

En Amérique du Nord comme en Europe, à l'origine du concept même d'intervention précoce on trouve le concept de risque. Des années quarante aux années soixante, la notion de risque associée au concept d'intervention précoce référait essentiellement à l'inférence d'un déficit de nature ontosystémique chez l'enfant (Sheehan, Snyder, \& Sheehan, 1999). L'enfant « à risque » était généralement un enfant présentant une déficience, d'origine connue, diagnostiquée sur le plan médical, ou d'origine inconnue, inférée, comme ce fut le cas avec l'hypothèse du retard scolaire précoce résultant de la dysfonction cérébrale minime (minimal brain damage). Si aucune déficience organique n'était identifiée, les difficultés scolaires et sociales étaient trop souvent attribuées à d'hypothétiques facteurs héréditaires (enfant d'alcoolique, de criminel, etc.).

Depuis une trentaine d'années maintenant, il est admis que l'enfant « à risque » d'origine biophysiologique représente une catégorie minoritaire, voire marginale, parmi les populations à risque de mésadaptation sociale et scolaire précoce. C'est plutôt l'inférence d'une forte probabilité de retard développemental et de difficultés d'intégration sociale et scolaire chez l'enfant de milieu socioéconomique faible (mséf) qui définit le risque. L'inférence d'un risque de sous-stimulation associée à la pauvreté, tout particulièrement en milieux ethniques, correspond à l'origine à la théorie du « déficit culturel » (Riessman, 1962). Cette dernière devint, à partir de la fin des années soixante, la source et la caution idéologique concomitante de multiples programmes d'intervention éducative plus ou moins massivement implantés dans les mséf, en particulier aux États-Unis (Coll, et al., 1996; Corcoran, 1995).

À l'origine de ces programmes s'adressant aux enfants de trois à cinq ans, se trouve le constat répété (Deutsch, 1963) qu'un nombre très élevé d'enfants en difficulté d'adaptation et d'apprentissage était issu des mséf.

Dans cette logique, l'enfant pauvre est considéré d'office en tant qu'enfant « à risque » (vulnérable). De façon générale, l'enfant vulnérable en est un dont soit les caractéristiques personnelles, constitutionnelles ou non, soit les caractéristiques environnementales, en particulier familiales, laissent appréhender dès le plus jeune âge une plus forte probabilité d'un développement psychopathologique que la moyenne des enfants de son âge dans un contexte social et culturel donné. 
L'apparition ou non des difficultés dépend de la nature des interactions entre ses caractéristiques individuelles et les caractéristiques de l'écosystème dans lequel il se développe. En nous référant à un modèle que nous avons déjà exposé lors de précédentes publications (Terrisse, Larose, \& Lefebvre, 1998; Terrisse \& Larose, 2003), nous situons le développement de l'enfant dans l'une ou l'autre des quatre conjonctures suivantes:

- l'enfant sans déficiences se développant dans un environnement favorable;

- l'enfant avec déficiences se développant dans un environnement favorable;

- l'enfant sans déficiences se développant dans un environnement défavorable;

- l'enfant avec déficiences se développant dans un environnement défavorable.

Les enfants «à risque» se situent majoritairement dans la troisième situation, (qui correspond à celle des enfants de mséf) puisque le taux de prévalence d'enfants présentant des déficiences pré et néo-natales est relativement peu élevé. C'est donc dans cette conjoncture que s'est développé la plupart des projets d'intervention socio-éducative précoce (Wright, Diener, \& Kay, 1998).

\section{Les concepts de «facteurs de risque » et de «facteurs de protection » dans une perspective écosystémique}

Les facteurs de risque sont définis par Garmezy (1985) et Masten, Hubbard, Gest, Tellegen, Garmezy et Ramirez (1999) comme des facteurs dont la présence accroît la possibilité qu'un enfant ait des difficultés d'adaptation et d'apprentissage s'il est comparé avec un enfant d'une population de la même origine. À l'inverse, les facteurs de protection sont définis, toujours par Garmezy (1985), comme des caractéristiques des individus et de l'environnement susceptibles de contrer ou de limiter les effets des facteurs de risque. L'étude des facteurs de protection implique donc que l'enfant soit confronté à des conditions défavorables. Tant les facteurs de risque que les facteurs de protection peuvent être reliés soit à l'individu, soit à son écosystème, ou plutôt aux interactions entre les différents niveaux de cet écosystème en constante mutation dans le temps (chronosystème) ainsi qu'aux interactions dans chacun des niveaux eux-mêmes (endosystèmes). Chaque niveau de l'écosystème peut générer des facteurs de risque mais aussi des facteurs de protection. En fait, 
c'est plutôt les interactions relevant de différents niveaux de l'écosystème qui peuvent conduire l'individu à une situation d'échec ou au contraire à croître ou à s'adapter malgré des circonstances adverses.

Ceci nous amène donc à tenter de préciser l'état des connaissances sur l'identification des principaux facteurs de risque et des facteurs de protection. Les recherches dans ce domaine se sont beaucoup plus intéressées aux caractéristiques de l'enfant lui-même puis à celles de son microsystème (surtout sa famille) et, à un degré moindre, à celles du mésosystème, qu'aux facteurs relevant de l'exo et du macrosystème. Ceci est sans doute dû au fait que les effets des facteurs inhérents à ces deux derniers niveaux sont moins directement apparents, plus abstraits et qu'ils sont surtout inférés.

En effet, les facteurs d'ordre macrosystémique (valeurs, idéologies, croyances) ne deviennent observables que lorsqu'ils se traduisent par des choix de société et des politiques gouvernementales puisqu'ils sont opérationnalisés dans des institutions. L'identification des facteurs de risque et des facteurs de protection a fait l'objet de plusieurs travaux dont ceux de Kimchi et Schaffner (1990) et de Grizenko et Fisher (1992). Nous avons complété ces travaux par une recension des écrits plus récents et nous avons récemment proposé un modèle de catégorisation où nous distinguons, d'une part, les facteurs individuels et, d'autre part, les facteurs environnementaux reliés aux différents niveaux de l'écosystème (Terrisse, 2000).

En bref, dans les facteurs individuels, nous distinguons les facteurs constitutionnels des facteurs acquis et, parmi ceux-ci, les facteurs organiques des facteurs psychologiques et, selon les différentes périodes, pré, péri, néo et post-natales. Dans les facteurs microsystémiques, nous distinguons dans l'environnement familial les facteurs socio-économiques des facteurs psychologiques et, dans le milieu de garde (crèche et autres milieux d'intervention éducative s'adressant aux enfants de la naissance à cinq ans), les facteurs structurels des facteurs psychosociologiques. Parmi les facteurs mésosystémiques, nous distinguons également ceux qui sont liés aux intervenants (psychosociologiques) de ceux qui sont de type structurel. En ce qui concerne les facteurs exosystémiques nous retenons les facteurs relevant des institutions et des services qui devraient refléter les choix issus du macrosystème. Enfin, en ce qui concerne les facteurs macrosystémiques il est évident que les valeurs de finalité d'existence (Justice, Égalité, Solidarité, etc.) ne peuvent être en soi considérées comme pouvant constituer des facteurs de risque ou des facteurs de protection dans nos sociétés. Nous ne retiendrons donc que les valeurs instrumentales qui se traduisent par des politiques et des lois. 
Nous devons rappeler toutefois que ces catégorisations sont toutes relatives. En effet, d'une part, les probabilités qu'un seul facteur de risque ou qu'un seul facteur de protection ait des effets sur l'adaptation de l'enfant sont faibles, ceux-ci étant dus à un cumul de facteurs et aux interactions de ces facteurs entre eux. D' autre part, la distinction entre facteurs constitutionnels individuels et facteurs environnementaux est également discutable. Ainsi, les facteurs individuels constitutionnels, même prénatals, sont également tributaires de l'environnement. Par exemple, les recherches ont mis en évidence les effets de la défavorisation socio-économique sur le développement du fotus: moins de surveillance médicale, absence de prévention, alimentation moins équilibrée, habitudes de vie inadéquates. La mère agit comme médiatrice entre l'enfant qu'elle porte et l'environnement, qui génère déjà des facteurs de risque pour l'embryon.

De plus, certains facteurs de risque ou de protection, certaines variables environnementales, peuvent être considérés de façon contextuelle comme faisant partie du microsystème ou du mésosystème. Leur classification dans l'un ou l'autre des niveaux de proximité environnementale de l'enfant est donc souvent toute relative. Par exemple, la fréquentation d'un milieu de garde peut être à la fois considérée en tant que facteur appartenant au microsystème ou au mésosystème. Ainsi, la fréquentation quotidienne, huit ou neuf heures par jour, d'un milieu de garde de type familial correspond à la présence d'un second microsystème pour l'enfant. Inversement, un milieu de garde «structuré», fréquenté à raison de deux demi-journées par semaine, pourra être considéré comme faisant partie du mésosystème.

Les facteurs de risque et les facteurs de protection ont été identifiés au cours de multiples recherches sur le jeune enfant (de la naissance à six ans) et sa famille dans diverses disciplines selon des approches autant cliniques qu'expérimentales, qualitatives que quantitatives, transversales que longitudinales. Toutefois, ces facteurs ne sont pas immuables car un facteur de protection peut se transformer en facteur de risque selon l'âge de l'enfant et l'évolution du contexte. Ainsi, la surveillance vigilante du nourrisson, facteur de protection, peut devenir un facteur de risque à quatre ou cinq ans (surprotection).

Il faut souligner aussi qu'il existe un effet de cumul tant des facteurs de risque que des facteurs de protection. L'existence d'un seul facteur de risque chez l'enfant ou dans son environnement ne permet pas d'appréhender une possibilité de difficultés ultérieures plus élevées, pas plus qu'un seul facteur de protection n'est susceptible d'expliquer son éventuelle résilience. Rutter (1985) mentionne que la présence simultanée de deux facteurs de risque multiplie par quatre la possibilité qu'il y ait des difficultés d'adaptation socio-affective chez 
un enfant et que cette possibilité augmente considérablement avec le nombre de facteurs de risque.

Werner et Smith (1989) ont aussi montré dans un échantillon de 698 enfants suivis lors d'une étude longitudinale, que ceux qui présentaient à l'âge de dix ans le plus de difficultés d'adaptation étaient ceux chez qui ils avaient identifié au moins quatre facteurs de risque à l'âge de deux ans car le cumul de ces facteurs suscite un niveau de stress élevé chez l'individu. En nous référant à Lazarus et Folkman (1984), nous définissons le stress comme un état de tension résultant d'interactions négatives entre l'enfant et son environnement. Il est confronté à des situations adverses que ses ressources personnelles ne lui permettent pas de contrôler et qui deviennent, de ce fait, menaçantes, d'où la probabilité que, dans sa recherche d'équilibre avec son environnement, il adopte des stratégies d'adaptation (coping) inadéquates se traduisant, par exemple, par des difficultés comportementales. Dans le cas contraire, si la mobilisation de ses ressources personnelles et de celles de son environnement lui permet de développer des stratégies adéquates et couronnées de succès, nous parlerons de résilience.

\section{Le construit de résilience}

Nous définissons la résilience comme la capacité d'un individu à s'adapter avec succès à un environnement (social, scolaire, professionnel...) malgré des conditions défavorables. Nous rejoignons ici les définitions de Wang et Haertel (1995) en retenant le concept d' " adaptation réussie » plutôt que celui d' « invulnérabilité » (Wedell, 1998). Ce concept nous semble mieux correspondre aux interactions complexes, dynamiques et évolutives entre les facteurs de protection et les facteurs de risque présents chez l'individu et dans son environnement, que le concept d' « invulnérabilité » qui nous paraît traduire un état inné et statique. Les facteurs de protection sont les facteurs dans l'écosystème d'un individu favorables à son adaptation sociale, scolaire et professionnelle alors que les facteurs de risque sont les facteurs défavorables. Ces facteurs chez l'enfant et dans son microsystème familial peuvent être:

- Inhérents au sujet (ontosystème) et d'ordre psychobiologique, par exemple le sexe, l'âge, l'origine ethnique, les caractéristiques physiques et neurologiques, etc.

- Propres à sa famille et d'ordre psycho-sociologique, par exemple les caractéristiques psychologiques des parents, leurs attitudes, représentations, pratiques, la structure familiale, la présence des réseaux sociaux de soutien, etc. 
- Propres également à sa famille mais d'ordre sociologique et anthropologique, par exemple le niveau de scolarité des parents, leur profession, leurs revenus; la permanence d'un emploi, le type d'habitat, etc.

Déjà, dans le milieu familial, la combinaison de facteurs de protection peut annihiler les effets des facteurs de risques. Ainsi, une scolarité élevée, associée à une grande disponibilité chez la mère et des représentations positives de la scolarisation peuvent contrer les effets de la pauvreté et de la monoparentalité. Il serait possible de dire ici qu'il y a «résilience» à des conditions de prime abord adverses. Mais ces combinaisons de facteurs favorables peuvent se retrouver dans les interrelations avec d'autres systèmes. Par exemple, dans la classe, des attitudes et des pratiques adéquates ainsi que des représentations positives de l'enfant et de son milieu de la part de son enseignant peuvent constituer des facteurs de protection contrant des facteurs de risques familiaux. Au niveau du mésosytème scolaire, à l'école, la présence de programmes adaptés, de services, de personnes-ressources peuvent avoir le même rôle.

Il est donc impossible de déterminer si certaines conditions spécifiques adverses peuvent engendrer les mêmes effets à tous les stades de

développement, de socialisation et d'apprentissage d'un enfant puisque ceux-ci sont les résultats d'interactions très complexes entre les facteurs de protection et les facteurs de risque chez l'enfant lui-même et dans son environnement. Le modèle structuro-comportemental d'Horowitz (1987) illustre bien cette complexité et souligne le fait que l'adaptation d'un individu dans des situations adverses, même si cette adaptation est réussie, aurait sans doute été différente dans un environnement favorable.

\section{La variabilité du construit de résilience dans les écrits}

La littérature scientifique identifie, grosso modo, trois dimensions au construit de résilience:

- les facteurs individuels prédisant ou favorisant la résilience chez l'enfant;

- les facteurs environnementaux agissant sur les probabilités de résilience chez l'enfant;

- les caractéristiques définissant l'individu résilient. 
À leur tour, ces trois dimensions correspondent à trois types d'études différentes reposant sur des postulats qui peuvent s'avérer, selon le cas, complémentaires ou divergents.

Le premier type d'études cherche à identifier des variables prédictrices d'une capacité de résistance ou d'adaptation aux conditions adverses (Baldwin, Baldwin, Kasser, Zax, Sameroff, \& Seifer, 1993; Conrad, 1998; Cowen, Wyman, \& Work, 1996; Smith \& Prior, 1995). En rupture avec les traditions de recherche d'orientation béhavioriste, ces études sont centrées sur l'objectivation des caractéristiques individuelles qui «protègeraient» l'enfant. Sans postuler de façon claire la nature intrinsèque ou innée de la «qualité » de résistance de l'enfant, ces études se situent généralement dans la perspective de l'«invulnérabilité».

Le second type d'études se situe généralement dans une mouvance associée à l'héritage de la tradition béhavioriste fondant l'intervention préventive auprès des populations dites « à risque ». À l'origine, ces recherches se centraient principalement sur l'identification des variables environnementales prédisant généralement la mésadaptation sociale et scolaire tout en tentant d'identifier de façon concomitante les variables qui peuvent réduire le risque induit. Ces dernières étaient cependant postulées comme étant absentes a priori du régistre d'attitudes ou de conduites de l'enfant. Il s'agissait donc souvent de recherches associées au développement de programmes de prévention, dont la population cible était relativement large (Battistich, Schaps, Watson, \& Solomon, 1996; Engle, Castle, \& Menon, 1996). Depuis quelques années cependant, ces recherches tendent à évoluer vers l'identification de structures d'interaction entre variables individuelles et environnementales prédisant la probabilité d'adaptation sociale et scolaire. Il y a donc glissement d'une centration sur le concept de facteur de risque vers celui de facteur de protection (Miller, Brehm, \& Whitehouse, 1998; Pianta \& Walsh, 1998; Smith \& Carlson, 1997).

Le troisième type d'études, enfin, plus marginal dans la littérature, vise l'identification des caractéristiques de la « résilience état». Les recherches qui s'y rattachent postulent de façon plus ou moins explicite que la résilience est le produit d'un processus d'adaptation de l'individu mais ne s'attardent pas à la description de ce dernier. Elles s'intéressent plutôt à la description des caractéristiques d'individus, le plus souvent mais non exclusivement adultes, qui ont su surmonter des circonstances adverses « graves » ou traumatisantes (Ajdukovic \& Ajdukovic, 1998). Le critère de sélection des sujets s'avère généralement la stabilité des conduites adaptées dans un contexte où les stresseurs ne sont plus présents (Chambers \& Belicki, 1998). 
Nos travaux s'inscrivent plutôt dans la deuxième tendance. Cependant, ils s'en démarquent partiellement dans la mesure où ils se fondent sur une perspective épistémologique résolument socio-constructiviste. Dans cette perspective, la résilience correspond à un état relatif, contextualisé. La construction des structures cognitives, affectives ou conatives caractérisant un état de résilience dépend étroitement de la nature des interactions sociales que l'individu entretient avec les différents univers qui constituent son environnement (Egeland, Carlson, \& Sroufe, 1993). Dans cette perspective, les contextes avec lesquels l'enfant interagit impliquent des processus d'adaptation spécifiques et particuliers. Il peut donc s'avérer résilient à un environnement déterminé, à un certain stade de son développement, mais développer des schèmes d'action ou des structures d'interaction sociale inappropriées dans un autre environnement à un même stade ou encore dans un contexte similaire à une autre étape de sa vie (Freitas \& Downey, 1998). Cependant, comme il aura intériorisé des schèmes d'action distincts, fonctionnels à une certaine étape de son développement ou dans certains contextes, il présentera aussi de meilleures chances de les utiliser ultérieurement en tant que compétences adaptables ou utilisables afin de modifier ses conduites ou d'en développer de nouvelles, plus adaptées au nouveau contexte.

\section{Les limites des études de résilience et de l'applicabilité d'une perspective écosystémique de type constructiviste au préscolaire}

Les études récentes menées auprès des populations d'âge préscolaire ou primaire provenant de populations de mséf, lorsqu'elles adoptent une perspective de résilience, tendent à souligner certains éléments qui font douter de l'application d'une réelle perspective écosystémique, tant au niveau de l'intervention précoce que de l'intervention éducative au primaire. Ces études tendent à suggérer que certaines mesures d'intervention éducative précoce ont peu d'effets observables qui leur soient directement imputables, sur l'adaptation scolaire et sociale à moyen terme des enfants qui en ont bénéficié (Terrisse, Larose, \& Lefebvre, 2001).

L'atténuation éventuelle de l'impact observé des mesures éducatives précoces sur le rendement et la persévérance scolaire des enfants provenant de mséf, dès la seconde ou la troisième année de scolarité primaire, fait l'objet de controverses soutenues dont fait état Barnett (1995). De nombreuses études menées récemment aux États-Unis dans le cadre de l'évaluation des effets des programmes de type «Head-Start » suggèrent que les effets de certaines mesures d'éducation préscolaire en mséf pourraient dépendre en partie de la nature transitoire ou, à l'inverse, durable et récurrente du statut de pauvreté dans lesquel 
se trouvent les familles des enfants bénéficiaires (McLoyd, 1998). Des études évaluatives récentes, menées au Québec sur l'impact des mesures d'intervention éducative précoce en mséf font un constat similaire (Terrisse, Lefebvre, et al., 2000).

C'est peut-être la raison pour laquelle les études de résilience portant sur les populations jugées à risque aux États-Unis, notamment lorsqu'elles se réclament d'une perspective écosystémique, tendent à se limiter aux souspopulations présentant une «culture» durable de la pauvreté. Ceci expliquerait la concentration des mesures d'intervention précoce, ainsi que du suivi de ses effets, auprès des populations afro-américaines ou hispano-américaines dont la définition ne se fait plus tellement sur le plan des caractéristiques ethnolinguistiques mais sur celle d'un statut de défavorisation endémique, durable, et de concentration territoriale.

D'une façon plus générale, la documentation scientifique suggère que les caractéristiques psychosociales ainsi que les conduites parentales pourraient agir en tant que variables modératrices sur la probabilité de résilience scolaire à long terme des enfants de mséf (Reynolds, Mavrogenes, Bezruczko, \& Hagemann, 1996). L'interaction entre ces caractéristiques et un ensemble de variables propres à l'intervention éducative au préscolaire ainsi qu'en scolarité régulière pourrait agir en tant que soutien à la motivation, à l'apprentissage et à la persévérance scolaire (Kinch \& Schweinhart, 1999; Larose, Bourque, Terrisse, \& Kurtness, 2001; Schweinhart \& Weikart, 1998). Au chapitre des variables modératrices qui, selon les plus récentes recherches, interagissent potentiellement avec les attitudes et pratiques éducatives parentales, mentionnons:

- le type de soutien au développement cognitif de l'enfant correspondant au profil particulier d'un programme éducatif au préscolaire;

- $\quad$ les représentations ainsi que les attentes d'efficacité de la part des intervenants qui accueillent les enfants provenant de mséf;

- les représentations ainsi que les attentes d'efficacité de la part des enseignants qui accueillent ces enfants lors de leur insertion en scolarité régulière;

- la cohérence entre les curriculums du préscolaire et ceux du primaire ainsi que celle qui devrait caractériser les pratiques enseignantes entre ces ordres d'enseignement.

L'instabilité des modèles d'intervention éducative véhiculés par les enseignants du préscolaire (maternelle 4 ans et maternelle 5 ans) ainsi que, tout 
particulièrement, du primaire, est un phénomène connu et documenté (Larose \& Lenoir, 1995; 1998; Lenoir, Larose, Grenon, \& Hasni, 2000; Schweinhart \& Weikart, 1998). Cette instabilité, ainsi que la variabilité des représentations que les enseignants partagent au regard du potentiel de succès et d'intégration scolaire et sociale harmonieuse des enfants provenant de mséf, constituent des facteurs qui affectent directement la probabilité que l'enfant puisse identifier des zones de cohérences entre ce qu'il vit au sein des divers microsystèmes dans lesquels il transite quotidiennement (Stipek \& Byler, 1997).

L'approche écosystémique, comprise dans une perspective non réductionniste et non comportementaliste, implique la prise en considération des interactions de l'enfant avec l'ensemble des composantes (acteurs et environnement physique) des systèmes au travers desquels il transite. Encore faut-il qu'il existe des zones d'interpénétration ou d'intégration des actions des acteurs de chacun des microsystèmes. Nous avons ici une première barrière à son application sur le plan de l'intervention. La majorité des études évaluatives portant sur l'intervention précoce auprès de l'enfant de mséf et de sa famille fait ressortir une contradiction majeure entre les perspectives officielles mettant de l'avant le partenariat intervenants-famille et la relation « expert-bénéficiaire » qui se manifeste dans la pratique (Terrisse, Lefebvre, et al., 2000). Pour leur part, les études qui portent sur la collaboration école famille, notamment lorsque celle-ci concerne les enfants provenant de populations jugées à risque, tendent à souligner deux phénomènes.

D'une part, les parents de mséf sont généralement sous-scolarisés. Plusieurs études évaluatives récentes suggèrent que l'implication scolaire parentale a plus d'impact chez les élèves qui proviennent de milieux socioéconomiques «moyens », où les parents ont des profils de scolarité plus stables, que chez ceux qui originent de populations de mséf (Shumow, Vandell, \& Kang, 1996), tout particulièrement lorsque les enfants vivent dans des structures familiales nucléaires «classiques» (Robinson, Weinberg, Redden, Ramey, \& Ramey, 1998).

Par ailleurs, les études qui portent sur la collaboration école famille tendent à suggérer l'existence de différences majeures d'attitudes et d'attentes entre les parents de mséf, leurs enfants et les enseignants au regard du rôle que les deux premières entités doivent jouer dans la dynamique scolaire (Crozier, 1997; Renaud \& Mannoni, 1997). Les enseignants ont généralement des attentes de type instrumental, les parents ne se sentent pas habilités dans ce domaine, et ce sentiment d'incompétence va croissant tout au long de la progression scolaire de l'enfant (Carlton \& Winsler, 1999).

D'autre part, les enseignants tendent à considérer leur intervention de façon cloisonnée dans le temps. L'ordre d'enseignement, le cycle 
d'enseignement à l'intérieur d'un même ordre sont perçus et traités, de facto, en tant que systèmes hermétiques les uns par rapport aux autres (Reyes, Gillock, Kobus, \& Sanchez, 2000; Rice, 2001; Rimm-Kaufman \& Pianta, 1999). L'apport des parents et leur collaboration en tant qu'agents éducatifs compétents est dès lors exclue dans ce contexte (La Paro, Pianta, \& Cox, 2000; Rimm-Kaufman, Pianta, \& Cox, 2000).

\section{Conclusion}

La popularisation du modèle écosystémique de Bronfenbrenner (1979) chez les chercheurs et les intervenants auprès de l'enfant de mséf et de sa famille a marqué un virage majeur dans la conception même de la défavorisation et de son impact sur des probabilités d'intégration sociale et scolaire harmonieuse. L'enfant n'est plus considéré en tant qu'individu exclusivement défini par le contexte où son action se manifeste, en tant qu'enfant à la maison, en tant qu'élève à l'école, mais plutôt en tant qu' acteur central au travers duquel les environnements, les systèmes dans lesquels il évolue, prennent un sens. Dans l'approche écosystémique, les systèmes n'ont d'existence que dans la mesure où ils sont une dimension spécifique du vécu de l'individu. Néanmoins, le rôle de l'enfant au sein de chacun de ces systèmes ainsi que sa capacité de les intégrer donc de les faire émerger en tant qu'entités sociales intégratrices, demeure sujet à variation selon le regard épistémologique que posent les adultes qui y oeuvrent ainsi que la perspective, développementale ou normative, qui guide la mise en oeuvre des finalités de chaque environnement d'accueil.

L'intervention écosystémique impose la prise en considération d'une complémentarité des acteurs et des finalités des systèmes qui composent l'environnement de l'enfant. Cette complémentarité, à son tour, suppose la reconnaissance de savoirs valides et de compétences réelles de la part de l'ensemble des acteurs de chaque système, à la fois chez leurs pairs ainsi que chez ceux qui appartiennent à un tiers « univers ». Ainsi ne suffit-il pas de transformer les curriculums (programmes dans l'acception européenne du terme) des environnements d'éducation préscolaire que fréquentent les enfants de mséf pour que les intervenants qui y oeuvrent considèrent la complémentarité de leur action et la spécificité ou la richesse de l'expérience que ces enfants y vivent. À plus forte raison ne suffit-il pas de proclamer la nécessaire articulation «famille-école » ou «famille-intervenants socio-éducatifs » pour que cette dernière fasse du parent de mséf un partenaire, un intervenant éclairé jouant un rôle positif en tant que gestionnaire du système qui, pourtant, aura longtemps la plus grande valeur affective pour l'enfant et, partant, la plus grande crédibilité. 
L'approche écosystémique en intervention éducative ne nous semble pouvoir prendre tout son sens que si elle se fonde sur une épistémologie constructiviste, voire socio-constructiviste, du développement humain. Dans une telle perspective, la recherche et l'identification des compétences plutôt que de «l'incompétence » des tiers-acteurs de l'univers enfantin comptent tout autant que celles des compétences construites par l'enfant lui-même. Ce primat de la compétence enfantine s'exerce par delà les risques qui pèsent sur son développement et par delà les conduites disfonctionnelles qu'il peut manifester au sein d'un système particulier, à un moment déterminé de son développement. C'est là l'essence même d'une définition fonctionnelle de la résilience sociale ou scolaire.

L'approche écosystémique peut caractériser l'intervention éducative précoce. En principe elle le fait déjà, ne serait-ce que par le poids du concept d'intervention de réseau dans ce domaine. Le défi consiste à créer les conditions qui permettent de passer d'une intervention réduisant la définition d'un écosystème à l'addition des caractéristiques propres aux dynamiques de chacun des systèmes où transite l'enfant, vers la définition d'un écosystème en tant qu'univers cohérent où nul acteur n'est spécialiste mais où tous les acteurs adultes sont des médiateurs entre l'enfant et des construits sociaux. Qu'est-ce que la famille, sinon une forme particulière de lieu de gestion d'interactions socialement déterminées? Qu'est-ce que l'école ou le milieu de garde, sinon des formes tout aussi particulières et tout aussi relatives, socialement déterminées, d'organisation d'espaces où est pratiquée la médiation entre le savoir construit au quotidien, celui de l'enfant, et le savoir homologué, socialement reconnu, celui de l'univers social ou scolaire?

Un tel « virage » semble possible. C'est tout au moins le sens que supposent les discours de réforme, tant des institutions de santé et de services sociaux que d'éducation qui prévalent actuellement dans la majeure partie des pays industrialisés. L'orientation officielle de ces réformes, au Canada ainsi qu'en Belgique et en Suisse, est officiellement socio-constructiviste. Reste à voir si nos sociétés, leurs écoles et leurs intervenants sociaux et scolaires s'y adapteront.

\section{Références}

Ajdukovic, M., \& Ajdukovic, D. (1998). Impact of displacement on the psychological well-being of refugee children. International Review of Psychiatry. 10(3), 186-195. 
Au, K. H. (1998). Social constructivism and the school literacy learning of students of diverse backgrounds. Journal of Literacy Research, 30(2), 297-319.

Baldwin, A. L., Baldwin, C. P., Kasser, T., Zax, M., Sameroff, A., \& Seifer, R. (1993). Contextual risk and resiliency during late adolescence. Development and Psychopathology, 5(4), 741-761.

Barnett, W. S. (1995). Long-term effects of early childhood programs on cognitive and school outcomes. Future of Children, 5(3), 25-50.

Battistich, V., Schaps, E., Watson, M., \& Solomon, D. (1996). Prevention effects of the Child Development project. Early findings from an ongoing multisite demonstration trial. Journal of Adolescent Research, 11(1), 12-35.

Bronfenbrenner, U. (1979). The Ecology of Human Development: Experiments by Nature and Design. Cambridge, MA: Harvard University Press.

Carlton, M. P., \& Winsler, A. (1999). School readiness: The need for a paradigm shift. School Psychology Review, 28(3), 338-352.

Chambers, E., \& Belicki, K. (1998). Using sleep dysfunction to explore the nature of resilience in adult survivors of childhood abuse or trauma. Child Abuse and Neglect, 22(8), 753-758.

Coll, C. G., Lamberty, G., Jenkins, R., Mcadoo, H. P., Crnic, K., Wasik, B. H., \& Garcia, H. V. (1996). An integrative model for the study of developmental competencies in minority children. Child Development, 67(5), 1891-1914.

Conrad, B. S. (1998). Maternal depressive symptoms and homeless children's mental health. Risk and resiliency. Archives of Psychiatric Nursing, 12(1), 50-58.

Corcoran, M. (1995). Rags to rags. Poverty and mobility in the United States. Annual Review of Sociology, 21, 237-267.

Cowen, E. L., Wyman, P. A., \& Work, W. C. (1996). Resilience in highly stressed urban children. Concepts and findings. Bulletin of the New York Academy of Medicine, 73(2), 267-284.

Crozier, G. (1997). Empowering the powerful. A discussion of the interrelation of government policies and consumerism with social class factors and the impact of this upon parent interventions in their children's schooling. British Journal of Sociology of Education, 18(2), 187-200.

Deutsch, M. (1963). The disadvantaged child and the learning process. New York: Columbia University, Teachers College Press.

Egeland, B., Carlson, E., \& Sroufe, L. A. (1993). Resilience as process. Development and Psychopathology, 5(4), 517-528. 
Engle, P. L., Castle, S., \& Menon P. (1996). Child development. Vulnerability and resilience. Social Science and Medicine, 43(5), 621-635.

Freitas, A. L., \& Downey, G. (1998). Resilience. Adynamic perspective. International Journal of Behavioral Development, 22(2), 263-285.

Garmezy, N. (1985). Stress resistant children: The search for protective factors. In J. Stevenson (Dir.), Recent research in developmental psychopathology (p. 213 - 233). Oxford: Pergamon Press.

Grizenko, N. G., \& Fisher, C. (1992). Review of studies of risk and protective factors for psychopathology in children. Canadian Journal of Psychiatry, 37(10), 711-721.

Holloway, S. D., Rambaud, M. F., Fuller, B,. \& Eggerspierola, C. (1995). What is appropriate practice at home and in child care. Low income mothers' views on preparing their children for school. Early Childhood Research Quarterly, 10(4), 451-473.

Horowitz, F. D. (1987). Exploring developmental theories. New York: Laurence Erlbaum Associates.

Howard, S., \& Johnson, B. (2000). What makes the difference? Children and teachers talk about resilient outcomes for children 'at risk'. Educational Studies, 26(3), 321-337.

Howard, S., Dryden, J., \& Johnson, B. (1999). Childhood resilience: Review and critique of literature. Oxford Review of Education, 25(3), 307-323.

Kimchi, J., \& Schaffner, B. (1990). Childhood protective factors and stress risk. In L.E. Arnold (Dir.), Chilhood stress (p. 475 - 500). New York: J. Wiley and Sons.

Kinch, A. F., \& Schweinhart, L .J. (1999). Making child care work for everyone: Lessons from the program recognition project. Young Children, 54(1), 68-92.

Laird, R. D., Pettit, G. S., Dodge, K. A., \& Bates, J. E. (1998). The social ecology of school-age child care. Journal of Applied Developmental Psychology, 19(3), 341-360.

La Paro, K. M., Pianta, R., \& Cox, M. (2000). Kindergarten teachers' reported use of kindergarten to first grade transition practices. Elementary School Journal, 101(1), 63-78.

Larose, F., Bourque, J., Terrisse, B., \& Kurtness, J. (2001). La résilience scolaire comme indice d'acculturation chez les autochtones. Revue des sciences de l'éducation, XXVII (1), 151-180.

Larose, F., \& Lenoir, Y. (1995). L'Interdisciplinarité didactique au primaire: Étude de l'évolution des représentations et des pratiques chez des titulaires du premier cycle du primaire dans le cadre d'une 
recherche-action-formation. (Rapports de recherche du LARIDD, no 2). Sherbrooke: Université de Sherbrooke, Faculté d'éducation.

Larose, F., \& Lenoir, Y. (1998). La formation continue d'enseignants du primaire à des pratiques interdisciplinaires: bilan de recherches et perspectives. Revue des sciences de l'éducation, XXIV(1), 199-240.

Lazarus, R. S., \& Folkman, S. (1984). Stress, Appraisal and coping. New York: Springer Verlag.

Lenoir, Y., Larose, F., Grenon, V., \& Hasni., A. (2000). La stratification des matières scolaires chez les enseignants du primaire au Québec: évolution ou stabilité des représentations depuis 1981? Revue des sciences de l'éducation, 26(3), 483-516.

Lenoir, Y., Larose, F., \& Hébert, M.-F. (2000). Analyse de quelques exemples d'utilisation du constructivisme au Québec dans les didactiques. Communication au colloque "Constructivisme et éducation », Université de Genève, 4-8 septembre. Document téléaccessible à l'URL: $<$ http://www.educ.usherb.ca/grife/membres/publications>

Lucyshyn, J. M., Albin, R. W., \& Nixon, C. D. (1997). Embedding comprehensive behavioral support in family ecology. An experimental single-case analysis. Journal of Consulting and Clinical Psychology, 65(2), 241251.

Masten, A. S., Hubbard, J. J., Gest, S. D., Tellegen, A., Garmezy, N., \& Ramirez, M. (1999). Competence in the context of adversity: Pathways to resilience and maladaptation from childhood to late adolescence. Development \& Psychopathology, 11(1), 143-169.

McLoyd, V. C. (1998). Socioeconomic disadvantage and child development. American Psychologist, 53(2), 185-204.

McMillen, J. C. (1999). Better for it: How people benefit from adversity. Social Work, 44(5), 455-468.

Miller, G. E., Brehm K., \& Whitehouse, S. (1998). Reconceptualizing school based prevention for antisocial behavior within a resiliency framework. School Psychology Review, 27(3), 364-379.

Miltenburg, R., \& Singer, E. (2000). A concept becomes a passion - Moral commitments and the affective development of the survivors of child abuse. Theory and Psychology, 10(4), 503-526.

Mortimer, J. T., \& Shanahan, M. J. (1994). Adolescent work experience and family relationships. Work \& Occupations, 21(4), Nov 1994, 369-384.

Ollendick, T. H., \& King, N. J. (1999). Child behavioral assessment and cognitive-behavioral interventions in schools. Psychology in the Schools, 36(5), 427-436. 
Palincsar, A. S. (1998). Social constructivist perspectives on teaching and learning. Annual Review of Psychology, 49, 345-375.

Pianta, R. C., \& Walsh, D. J. (1998). Applying the construct of resilience in schools. Cautions from a developmental system's perspective. School Psychology Review, 27(3), 407-417.

Renaud, L., \& Mannoni, C. (1997). Parental participation in curricular and extracurricular activities. Canadian Journal of Public Health / Revue Canadienne de Santé Publique, 88(3), 184-191.

Rey, B. (1996). Les compétences transversales en question. Paris: Esf.

Reyes, O., Gillock, K. L., Kobus, K., \& Sanchez, B. (2000). A longitudinal examination of the transition into senior high school for adolescents from urban, low-income status, and predominantly minority backgrounds. American Journal of Community Psychology, 28(4), 519-544.

Reynolds, A.J., Mavrogenes, N. A., Bezruczko, N., \& Hagemann, M. (1996). Cognitive and family-support mediators of preschool effectiveness. A confirmatory analysis. Child Development. 67(3), 1119-1140.

Rice, J. K. (2001). Explaining the negative impact of the transition from middle to high school on student performance in mathematics and science. Educational Administration Quarterly, 37(3), 372-400.

Riessman, F. (1962). The culturally deprived child: A new view. New York: Columbia University, Teachers College Press.

Rimm-Kaufman, S. E., \& Pianta, R. C. (1999). Patterns of family-school contact in preschool and kindergarten. School Psychology Review, 28(3), 426438.

Rimm-Kaufman, S. E., Pianta, R. E., \& Cox, M .J. (2000). Teachers' judgments of problems in the transition to kindergarten. Early Childhood Research Quarterly, 15(2), 147-166.

Robinson, N. M., Weinberg, R. A., Redden, D., Ramey, S. L., \& Ramey, C. T. (1998). Family factors associated with high academic competence among former Head-Start children. Gifted Child Quarterly, 42(3), 148156.

Rosenbaum, R., \& Dyckman, J. (1995). Integrating self and system. An empty intersection. Family Process, 34(1):21-44.

Rutter, M. (1985). Resilience in the face of adversity: Protective factors and resistance to psychiatric disorders. British Journal of Psychiatry, 147, 598-611.

Sheehan, R., Snyder, S., \& Sheehan, H. (1999). Les tendances de l'intervention précoce au début du 21e siècle aux États-Unis. In F. Peterhander, O. Speck, G. Pithon, 7 B. Terrisse (Dir.), Les tendances actuelles de 
l'intervention précoce en Europe (p. 165 - 180). Sprimont: Pierre Mardaga éditeur.

Shumow, L., Vandell, D. L., \& Kang, K. (1996). School choice, family characteristics and home-school relations. Contributors to school achievement. Journal of Educational Psychology, 88(3), 451-460.

Smith, C., \& Carlson, B. E. (1997). Stress, coping, and resilience in children and youth. Social Service Review, 71(2), 231-256.

Smith, J., \& Prior, M. (1995). Temperament and stress resilience in school-age children. A within families study. Journal of the American Academy of Child and Adolescent Psychiatry, 34(2), 168-179.

Sontag, J. C. (1996). Toward a comprehensive theoretical framework for disability research. Bronfenbrenner revisited. Journal of Special Education, 30(3), 319-344.

Stipek, D. J., \& Byler, P. (1997). Early childhood education teachers. Do they practice what they preach? Early Childhood Research Quarterly, 12(3), 305-325.

Stoiber, K. C., \& Good, B. (1998). Risk and resilience factors linked to problem behavior among urban, culturally diverse adolescents. School Psychology Review, 27(3), 380-397.

Schweinhart, L. J., \& Weikart, D. P. (1998). Why curriculum matters in early childhood education. Educational Leadership, 55(6), 57-72.

Terrisse, B. (2000). The resilient child: Theoretical perspectives and review of the literature. Ottawa, ON: Conseil des ministres de l'éducation du Canada. Pan-Canadian Education Research Agenda Symposium (PCERA2000). Simon Goldberg Conference Center, 6-7 avril 2000. http:/ /www.cmec.ca/stats/pcera/symposium2000

Terrisse, B., \& Larose, F. (2003). L'intervention socio-éducative précoce auprès de la famille. In Ministère de l'éducation nationale, de la formation professionnelle et des sports (dir.). Actes du colloque "Difficulté d'apprendre, difficulté d'enseigner ». Luxembourg (Grand-Duché): Ministère de l'Éducation Nationale, de la Formation Professionnelle et des Sports. Document téléaccessible en format .pdf à http:// www.script.lu/activinno/conferences_difficulte_apprendre/ conferences_difficulte_apprendre_terrisse_texte-continu.pdf

Terrisse, B., Larose, F., \& Lefebvre, M. L. (1998). L'évaluation des facteurs de risque et de protection dans la famille: développement et validation du Questionnaire sur l'environnement familial. La revue internationale de l'éducation familiale, recherches et interventions, 2(2), 39-62.

Terrisse, B., Larose, F., \& Lefebvre, M. L. (2001). La résilience: facteurs de risques et facteurs de protection dans l'environnement social et scolaire du jeune enfant. Cahiers du Centre de recherche sur les 
formes d'éducation et d'enseignement, 16, 129-172 (no thématique, École/Famille:quelles médiations?)

Terrisse, B., Lefebvre, M.-L., Larose, F., \& Martinet, N. (2000). Analyse des caractéristiques des mesures d'intervention éducative précoce et comparaison de leurs effets à moyen terme sur l'adaptation scolaire et sociale d'enfants de milieux socio-économiquement faibles. Rapport final de recherche présenté au Conseil québécois de la recherche sociale pour la subvention no. 2677095. Montréal: UQAM, Département des sciences de l'éducation.

Tyler, K., \& Jones, B. D. (2000). Implementing the ecosystemic approach to changing chronic problem behaviour in schools. Educational Psychology, 20(1), 85-98.

Waller, M. A. (2001). Resilience in ecosystemic context: Evolution of the concept. American Journal of Orthopsychiatry, 71(3), 290-297.

Wallon, H. (1938). La vie mentale. L'Encyclopédie française, Vol. VIII. Paris: Presses universitaires de France.

Walsh, F. (1996). The concept of family resilience. Crisis and challenge. Family Process, 35(3), 261-281.

Wang, M. C., \& Haertel, G. D. (1995). Educational resilience. In M. C. Wang, M. C. Reynolds, \& H. J. Walberg (Dir.), Handbook of special and remedial education. Research and practice (p. 159 - 204). New York: Pergamon Press (2e édition).

Wedell, K. (1998). L'intervention compensatoire: un modèle explicatif de la vulnérabilité et de l'invulnérabilité aux facteurs environnementaux défavorables chez des enfants ayant des problèmes spécifiques. In $\mathrm{F}$. Peterander, O. Speck, G. Pithon, \& B. Terrisse (Dir.), Les tendances actuelles de l'intervention précoce en Europe. (p. 235 - 247). Sprimont: Pierre Mardaga.

Werner, E. E., \& Smith, R. S. (1989). Vulnerable but invincible: A longitudinal study of resilient children and youth. New York: Adams-Whister-Cox. 